\title{
The use of the intranet of the Durban University of Technology Library as a Knowledge Management tool
}

\author{
David Thomas ${ }^{1}$ and Peter G. Underwood ${ }^{2}$ \\ davidt@dut.ac.za ORCID: orcid.org/0000-0002-3292-6014 \\ pgunderwood@wol.co.za ORCID: orcid.org/0000-0002-3292-6014
}

\begin{abstract}
Received: 2 February 2015
Accepted: 3 July 2015

This paper reports an investigation of the use of the Durban University of Technology (DUT) Library intranet as a knowledge management (KM) tool. The general objectives of the investigation were to establish what KM practices are used in the library of DUT; establish if and how the staff of this academic library employ the intranet as a tool for KM; identify the characteristics of a KM tool that could assist the management of the library; assess DUT Library's intranet (Microsoft SharePoint) against the characteristics of a KM tool. The specific objective was to ascertain the extent to which it is being used for KM and to develop an understanding of the factors that contribute or could contribute to the adoption of it for KM. The target population for this study was DUT professional library staff. The study employed a qualitative approach in its research design. Data were collected from the staff by means of a self-administered questionnaire, a focus group interview with a sample of key informants, and by consulting usage statistics of the SharePoint application.
\end{abstract}

Keywords: Microsoft SharePoint, Durban University of Technology, Knowledge Management, intranets, user studies

\section{Introduction and background}

Universities play a pivotal role in the knowledge society as proponents of knowledge production and dissemination (Beerkens 2008: 17). Drucker, cited by Beerkens (2008: 17), suggests that the key to growth is to work smarter rather than harder. It is this very principle that drives Knowledge Management (KM). In a knowledge society, knowledge plays a key role in ensuring development through harnessing the wealth of knowledge that is in existence and building new knowledge based on it. Perez (1999) suggests that there are various tools and technologies that can enable KM; consequently, it was decided to investigate the extent to which the intranet of the library of the Durban University of Technology (DUT) could be used as such an enabling tool.

\section{Statement of the problem}

DUT Library, like many others, has witnessed high staff turnover, necessitating a continuous, and expensive, programme of staff training to replace skills. Even so, this does not ensure that all aspects of organisational knowledge are retained or passed on: there is a need for other avenues to be explored in tapping into the library's knowledge base and ensuring that such knowledge assets are not lost. Hence, the results of this investigation, which sought to assess whether SharePoint, the intranet system used by DUT Library, could serve as a knowledge management tool. The study focused on understanding to what extent DUT Library is using its intranet for KM.

\section{Literature review}

Higher education institutions are facing similar, if not the same, pressures as those experienced in the corporate sector, having to remain ahead of the competition (Yaying 2005: 36). Rowley (2000: 325) contends that higher education institutions need to be able to 'innovate or die' in response to marketplace pressures. This implies that they need to learn and adapt in the context of an ever-changing global information society. One of the ways to achieve this is by being able to recognise the knowledge assets already at their disposal, recognising the value of these assets and facilitating access to it. Effective management of these knowledge assets manifests itself in the products and services offered by DUT Library, among the many services supplied by the university overall. Access to, as well as consistent and effective use of, knowledge assets can position DUT favourably in relation to the academic job market and the market for student enrolment.

\subsection{Knowledge management}

'Knowledge management' refers to processes or activities that are geared toward managing those knowledge assets of the organisation that contribute toward adding value, facilitate strategic planning and highlight effective tactical choices

1. David Thomas is a Master of Information Technology (M.IT) graduate, University of Pretoria.

2. Peter G. Underwood is a M.IT Supervisor, University of Pretoria. 
(Sarrafzadeh, Martin \& Hazeri 2010: 199). KM ultimately aims at identifying what relevant knowledge is available through a knowledge audit; identifying where it resides; creating an environment conducive for its sharing and capturing; and employing mechanisms and tools that codify and store it in a manner that it could be accessed in order to generate new knowledge (Yaacob, Jamaluddin \& Jusoff 2010). Averweg and Jory (2007: 5) suggest that KM involves gaining business insights from that knowledge wherever it may reside, whether in documents or with the employees, and cultivating and establishing relationships between this content to offer corporate value.

Information and Communication Technology (ICT) has allowed for the development of tools that enable the flow of knowledge that is critical in the global information society. KM tools vary in form and function and therefore particular consideration needs to be given to the purpose and the appropriateness of the tools, within the context of an organisation. Balmisse, Meingan and Passerini (2007: 120) have identified four functional requirements for KM tools:

a. Facilitation of information contextualisation

In order to facilitate the discovery and management of information, metadata is one feature that can add value and contextualises the knowledge in a given setting. The metadata describes the content and its context and thereby facilitates its discovery: it should be added to content;

b. Intelligent transfer of information

Knowledge recovered from the knowledge database must meet the user's needs. It should be user-specific or contextually personalised according to the need. The KM tool must be able to accommodate in a balanced manner the user's needs, the nature of the content and the desired timing of transfer;

c. Facilitation of social interaction and networking

Digital socialisation attempts to facilitate social engagement through creating an enabling online environment that allows for and embraces social networking and engagement facilities. It must encourage and facilitate a mixture of spontaneous and more co-ordinated engagement using social networking facilities that allow both synchronous and asynchronous communication;

d. Customised human-computer interface

The quality of the interface that a system provides may determine whether individuals return to reuse the system or are deterred from further use. It implicitly contributes to the success of the system and, irrespective of how strong or good the database or search engine may be, users tend to judge the system based on their experiences with the front end or user interface.

\subsection{Intranet and Knowledge Management}

In enabling the sharing of knowledge, the provision of an appropriate tool is essential. Mphidi and Snyman (2004: 395) support the sentiments of Guenther (2003: 66) in describing intranets as similar to the human body's circulatory system in that an intranet connects the different parts of the organisation by facilitating an easier, more effective, flow of information within the organisation. The analogy is quite apt in highlighting an important enabler of knowledge sharing, which is connectedness. Knowledge sharing requires appropriate channels that would communicate that knowledge. An intranet should have embedded technologies or functionalities that allow for such sharing to occur, in order for it to be considered a tool for knowledge sharing.

Fenz (2012: 240) suggests that intranets are changing the way an organisation creates, processes, receives and disseminates information: an intranet may be understood as the internal web of an organisation that is built on the same protocols and technologies as that of the internet but with the fundamental difference being that the intranet focuses on an internal audience and content, thus supporting various activities within the organisation that include document management, communication and collaboration, even across geographically-dispersed sites (Fichter 1999: 107). Intranets should allow for multiple platform capability and security controls relative to the needs of the organisation. Although the intranet operates on the same backbone as the internet protocols, by its very nature, it is owned and managed by the organisation and it is the organisation that controls what content is to be made accessible and to whom (Edenius \& Borgerson 2003: 124). Further, intranets can be built to meet the needs of an organisation using various proprietary or open-source software packages that are available.

\subsection{SharePoint as an intranet}

If priority is given to maintaining and updating publicly-accessible websites and the content of intranets is, by comparison, neglected, outdated content will be the result. Good design principles suggest that there is a need for formal arrangements for updating if such intranets are to be found useful. With the growth of enterprises and the increased focus on knowledge assets within the organisation there has developed a need for a more robust system that could facilitate communication, access to information and a shared responsibility for amending content. There are several software solutions that offer more than the traditional intranet functionality by including portal, web content management and business intelligence capabilities. SharePoint is one such solution that has received, and continues to receive, attention as a solution (Koplowitz \& Owens 2009).

SharePoint as a web-based collaboration and document management platform has enjoyed growth in its adoption since its launch in 2001 (Jedd 2009: 34). Weldon (2010) further confirms that SharePoint started off as an IT-only application but has evolved to the point that it now requires input from both the installers and the users. Those who install the application and those that will be using it need to collaborate to establish how SharePoint should be set up to fit the requirements of the organisation. As with most enterprise software applications, a degree of customisation is available 
and possible through collaboration between stakeholders. Herrera (2008: 87) describes SharePoint as a platform for collaboration and communication that incorporates a suite of communication and collaboration tools, Web 2.0 technologies as well as other added features for online surveys, project management and even image libraries.

The first iteration of the intranet model allowed only authorised, specifically trained or skilled individuals to upload and update content at their convenience. This restriction was soon abandoned with the development of applications such as Lotus Notes; other content management systems with web capability were quick to develop distributed publishing systems operating within restricted domains, and designed with the ease of use previously encountered with 'WYSIWYG' word-processing applications. SharePoint, following this trend, allows for the empowerment of the general user who can publish content directly without any intermediary or specialised skills. The flexibility of SharePoint has allowed for the user to engage directly with the platform with little technical knowledge (Herrera 2008).

In describing the nature of the platform, SharePoint has been described as a 'Swiss Army knife': it has multiple functions all rolled up into one compact platform (Jedd 2009: 35). As a product that features within the Microsoft Office suite, it should provide an integrated experience that allows the user to navigate easily between the suite components as well as the added convenience of a single sign-on to these Microsoft products. It does, however, mean that the organisation should be supporting and subscribing to a Windows network backbone and thus locking it in to use of a proprietary operating system (Jedd 2009: 35). Practitioners like Weintraub, as cited in Jedd (2009:36), caution that SharePoint imposes limitations on file sizes, and the number of documents in a library, together with other limitations associated with metadata, thesaurus and taxonomy management. It is further noted that some organisations prefer 'best of breed' products as opposed to opting for a SharePoint system that may offer relatively limited functionality. Installations have seen SharePoint adopted in conjunction with other enterprise content management systems to supplement SharePoint's limitations (Jedd 2009:35).

\section{Research method}

This study involved assessing the degree of understanding of KM by DUT Library staff, the KM practices that were in place in DUT Library and to what extent the library intranet was used for this purpose. To achieve this, a survey questionnaire was administered to DUT Library staff. A further focus group interview was conducted with a sample of this population to correlate and to delve further into pertinent issues. The third data collection method involved consulting DUT Library intranet usage statistics, which gave an indication from the system as to the frequency and extent of usage. The research strategy employed was a case study with DUT Library being the object of the study. Case studies, also referred to as idiographic research, are employed when one needs to learn more about a phenomenon or situation that may not be adequately understood (Leedy \& Ormrod 2013: 141). Case study research allows the researcher to understand the behavioural patterns of the phenomenon being studied and even trace its relationship with other factors (Kothari 2004: 116).

\subsection{Research setting}

The study was conducted at DUT Library, which is located in the KwaZulu-Natal province in South Africa. The study population included thirty-three of the eighty-one permanently employed staff members at DUT Library. The forty-eight staff members who did not form part of the study group included those who function in library support services: namely the stack attendants, circulation desk staff, materials processing staff, administrative assistants and the researchers; the researchers excluded these staff members because none of them were users of SharePoint and could not be expected to have operational experience of the application. It was decided to limit the survey group to the thirty-three staff members who might be expected to have some experience of SharePoint because of their job roles. All thirty-three were sent the questionnaire, whilst a sample of twelve was drawn from this group for the focus group interviews. The sampling method employed was purposive sampling, which relied upon selecting a few informants who were known to have more extensive experience of using SharePoint and might reasonably be expected to have formed opinions about the application. Purposive sampling also gives the researcher better control over the variables involved (Singh 2006: 91). A further advantage is the cost and time saving associated with this type of sampling (Kothari 2004: 59).

\subsection{Data collection}

There were three data collection instruments used, the rationale being to establish patterns through the process of triangulation. Triangulation entails using multiple sources of data that may reveal correlations between the data being collected, contributing to the validity of the findings (Leedy \& Ormrod 2013: 102). The data collection methods employed in this study were the online self-administered questionnaire, a focus group interview and the analysis of statistical usage data from the SharePoint administrative module. A self-administered questionnaire was adopted as the method of data collection because it allowed the researchers easy access to the respondents, who are geographically dispersed across six sites. It also allowed for quick turnaround of data collection. The survey captured data from the selected population group about the extent to which they used the intranet at DUT Library for knowledge management practices. The semistructured questionnaire contained a combination of open-ended and closed questions. The questionnaire was pretested to eliminate any leading questions and to ensure neutrality while providing a context that clarified the scope of the study. An online survey tool, SurveyMonkey, was employed to administer it. One focus group interview was included: data that may not have surfaced during a one-on-one interview, may surface through the group engagement. The moderator or facilitator may opt to explore these issues further depending on the value that it offers to the study (Leedy \& Ormrod 2013: 
154). The researchers also reviewed the usage statistics generated by the system. The statistics provided a glimpse into the frequency and extent to which SharePoint was being used by the library staff.

\section{Findings}

Findings of the investigation are discussed below.

\subsection{Age and experience of population at DUT Library}

While it is acknowledged that DUT is a relatively young institution, formed in 2002 through the amalgamation of ML Sultan Technikon and Technikon Natal, the research has revealed that there is an almost even spread of newcomers and more seasoned staff within the library. This relative lack of an extensive institutional history highlights the necessity for the library management to harness the knowledge, expertise and experience of both contingents to position the library strategically.

\subsection{Understanding of Knowledge Management}

Participants in the study demonstrated a good understanding of the concept of KM and the benefits that it held for the organisation. Staff recognise KM as practices that include the capturing, storing and sharing of organisational knowledge that would benefit the organisation by ensuring knowledge retention. It is understood to include the harnessing of both tacit and explicit knowledge. Respondents see $\mathrm{KM}$ as beneficial to the library in two broad areas, namely staff empowerment and development, and improvements to its services. The empowerment of staff may result in a reduction of job-specific training, as the knowledge and experience of others may be accessible in various forms. Library services can become more efficient as access to the knowledge will facilitate more informed and innovative decision-making. On the whole, KM allows the organisation to offer an improved service by empowered staff, who benefit from access to the shared corporate knowledge, thereby saving organisational resources and ultimately contributing to the competitive advantage that the organisation may enjoy.

\subsection{Knowledge Management practices at DUT}

Concerning what KM practices were in place at DUT Library, there were twenty-three (67\%) responses which indicated that, although there were initiatives in place, they were a mixture of both formal and informal practices. There were also respondents who admitted that there were no initiatives in their functional area or that they are unaware of any. This suggests that either some sections have adopted and practice KM more than others or that there is a lack of awareness. Responses indicated that there are efforts to capture knowledge from retiring staff and these may be a combination of exit interviews, succession plans, coaching and mentoring initiatives, as well as retirees documenting their own expertise. Thirteen (40\%) respondents reported that they were unaware of efforts in this regard. Seventeen (52\%) respondents indicated that there were efforts at capturing knowledge as an on-going exercise that forms part of the normal job routine. There were a few responses that noted specific initiatives that contributed to KM, in the form of Communities of Practice, document repository activities, as well as job rotation. A general comment was that in some instances, lessons learnt were documented and shared with management rather than the rest of the staff.

There have been a few instances where respondents indicated that they were unaware of what practices were in place. Whilst it is understandable that practices may vary relative to the functional area, it may benefit the entire library if the experiences of these initiatives were shared. It also became evident that as a result of the study, some respondents became aware that activities that they were engaged in constituted KM, while others became acutely aware that there was so much more that could be done. The researchers are therefore of the opinion that, while staff may have the theoretical understanding of KM, its practical application needs to be understood in the context of the purpose and value that it offers. A mechanism that may facilitate the adoption of KM at DUT is purposeful communication between library management and staff on the possibilities that KM offers for both the organisation and the individual.

\subsection{Inhibiting factors related to Knowledge Management practices}

None of the respondents consider knowledge management activities as a threat to their employment; however nineteen $(58 \%)$ claim that time constraints prevented them from being involved in such activities. Other factors included workload, no acknowledgement for sharing, no reciprocal benefit and no clear direction. The assertions of Aswath and Gupta (2009: 188) support these findings, which identify these aspects as inhibitors to KM at the workplace. They further assert that there may be other underlying issues, such as the organisational culture, which also surfaced as a finding in this study. Other inhibiting factors that emerged are the absence of a policy and the lack of a sharing culture.

Arising from the study, it is a recommendation that a formal KM strategy be developed and that measures be put in place that demonstrate the commitment of DUT Library management to the process by establishing practices that facilitate development of a sharing environment.

\subsection{SharePoint platform}

The usage statistics, as well as feedback from the respondents, indicated that DUT implementation of SharePoint is not being used to its full extent or by all staff. Findings also indicated that the SharePoint application itself contributed to its poor usage. Five respondents (15\%) rated the SharePoint platform as excellent, thirteen (39\%) rated it as good, seven $(21 \%)$ rated it as average, one $(3 \%)$ as poor and yet another respondent $(3 \%)$ as inadequate. 
Lindvall, Rus and Sinha (2003: 144) assert that having a user-friendly interface can be a determining factor in ongoing use. According to the respondents of this study, the SharePoint interface is not very user friendly. The degree or extent to which one could customise or personalise the interface may be rather limited, as is usually the case with proprietary systems like SharePoint. While SharePoint does allow for a degree of interface configurations, customisation of the software is not as easy to achieve. Such systems allow for a certain latitude where a client can make minor changes; however, anything beyond this may require either a version upgrade or specific programming changes, which may not always be feasible or affordable. One needs therefore to consider whether such customisations are essential or a 'nice to have'. Manoeuvring through the site is not necessarily obvious to the average user. Some functionalities or options remain 'hidden' within subpages or tabs that may not be clear to the user and so these tend to be missed out. Navigating the system effectively is aided by proper navigation structure and information architecture. Many respondents reported that, due to a bad experience with SharePoint, typical of which was not being able to find where a button or feature resides, caused them to have a mental block toward the system and a tendency not to use it unless forced to do so.

Focus group participants felt that a further spin-off of use is that much of the activity on SharePoint was directed to professional staff and, as a result, excluded other staff. In addition to this perceived exclusivity, further related aspects also emerged. There is an array of different content housed on the library's intranet; however, respondents felt that there was a need for better organisation and maintenance of the content. These two aspects have served as a source of confusion and frustration in that staff could not find the content they were searching for or believed that the content was outdated. Koplowitz and Owens (2009: 8) stress that the accessibility, security, governance and distribution of content is affected by the manner in which it is structured and stored. Furthermore, uploading content onto SharePoint proved difficult. These sentiments were echoed by participants in the focus group commenting that SharePoint is particular about the size and versions of documents, thereby restricting certain activities.

Aswath and Gupta (2009: 188) confirm that content needs to be organised in a manner that aids its discovery. Respondents felt that content arrangement required attention. One of the layers of making content more discoverable is that of adding metadata or using a taxonomy. Metadata, or 'data about data', describes the content in a manner that clarifies and contextualises the content such that the user is guaranteed relevant content. Lindvall, Rus and Sinha (2003: 141), suggest that a taxonomy allows for knowledge mapping to take place and correlations between content to be established, which aids in the discovery of content. It is not clear as to whether the version of SharePoint being used allows for these features or to what extent they can be achieved, which reiterates the necessity for training.

Another aspect of information organisation would relate to how the content is listed within the repository list. Is the file naming structure consistent with other systems? Can files be renamed or deleted? Can the lists be formatted or rearranged according to user preference. Currently the only individual that is aware of these issues is the Library IT administrator who is the custodian of the library SharePoint installation.

A search engine is a critical component of information retrieval. Ideally, an intranet search engine should be able to search across all content and render a customised result based on the user preference. Ennis and Tims (2010: 8) and Weldon (2010) describe the search capability in SharePoint as quite robust and customisable: however this study revealed that not all respondents were clear as to whether SharePoint had a search engine built into it or how effectively it functioned. Currently not all respondents had adequate knowledge of this feature to judge the extent or depth to which the content was searched.

Although the alerting service of SharePoint functions like a traditional alerting service in terms of notifying the user of new discussion postings, participants in the focus group found the task of setting alerts for every activity tedious. The email system has proven to be a more robust system in this regard, as the user is informed automatically about incoming messages and appointments.

Herrera (2008: 87) highlights the capability of the discussion tool in SharePoint for asynchronous communication. It is this very issue that prompted a poor assessment of the discussion tool, in that it did not have the capability for synchronous communication. Respondents maintained that the discussion feature, which showed minimal activity, was a bit cumbersome in the layout, the alerts and editing options. It has browser compatibility issues in that it could not function properly with any browser other than Microsoft Internet Explorer. The discussion feature on SharePoint was not very well used. While this may again allude to the necessity for training, the limited activity may also relate to the sharing culture at the library. As was already mentioned, staff tend not to share or participate and so this may not necessarily be related to the tool used for discussion.

As with all innovation, the full potential of the SharePoint system can only be realised if users are aware of what it has to offer and how to use the features available. This important aspect has emerged both from the self-administered questionnaire as well as from the focus group interview. Respondents were clearly unaware that certain functionalities where available within the platform, pointing to the need for training. Additionally, the intranet is not visible enough, according to respondents. Respondents have alleged that the website address for the site was too complicated to remember and, unless it is bookmarked in their individual web browser, they would not be able to find the site. Whilst this may relate to configuration issues, the researchers cannot discount the impact of the mental block mentioned earlier.

Given the nature of the content stored on the intranet, it is understandable that there needs to be some security measures in place such as user authentication and content encryption. Such mechanisms confirm access and permission relative to the staff member's user profile and accordingly allows access based on the access rights assigned. Respondents have expressed their frustration at having to authenticate numerous times within a single session. Respondents also alleged that SharePoint lacks integration with other applications. It should be able to integrate 
seamlessly with not only Microsoft programs but also with other library programs, such that the user is not burdened with launching an external program to continue an activity. The researchers recognise that these issues may not necessarily be shortcomings of SharePoint, but rather that its applications and functionalities were not exploited or implemented in totality. Posting on the discussion forum is tagged to the profile of the person who made or responded to the post. Whilst this has value in that one can identify who is making the comment, it also serves as a deterrent in that staff may feel intimidated about posting comments. Although one can argue that a degree of professional maturity is necessary, this is a reality that has been reported and needs to be addressed.

SharePoint, according to respondents, is not a straightforward system. There are certain technical intricacies that one needs to be aware of to exploit the system fully. Reports about the document collaboration feature, for example, indicate that the system requires that the document be withdrawn ('checked out') before it is edited, and then needs to be uploaded again. Other features within the system also require technical knowledge that will allow for their effective use, highlighting further the need for training.

\section{Recommendations of the study}

Based on the findings and conclusions, several recommendations can be made.

There is a need for training on the use of SharePoint. Training would not only provide staff with the knowledge of what features and functionalities are available within the system but also how to use these features. The poor adoption of the tool may be partly attributed to staff not knowing what was available and how to use it. The training needs to include the technical knowledge necessary and should be a structured training programme that is staggered to prevent information overload. Furthermore, training staff on the use of the system may not necessarily get them to use it. Opportunities and time must be allowed for staff to either practise or participate in this environment. Driving visitor traffic to the site is only one part to 'converting' staff to adopt the tool. The site must offer a valuable experience. This may be achieved by ensuring that:

- $\quad$ there is relevant and up-to-date content hosted;

- the content is arranged in a logical systematic manner;

- $\quad$ the discussions are current, qualitative and inviting;

- $\quad$ old content is archived, yet kept available;

- a blended approach is adopted in capturing and delivering content, perhaps through the engagement of both multimedia and print-based mediums; and,

- $\quad$ enhancing and enabling technologies are incorporated.

There is a need for better management and administration of the site. In keeping with the purpose of knowledge sharing, perhaps more staff could be empowered with skills and access to maintain aspects of the site. Currently this responsibility resides with one individual. Section coordinators should take an active role in promoting the use of the tool by uploading relevant section content and encouraging engagement with the tool by embedding the routine workflow processes into the platform where possible. Relevant parties should investigate the extent and degree of customisation that is possible and then apply as is appropriate. The idea of rebranding the site is a good one; however cosmetic changes may not be sufficient. The site may need a complete overhaul that addresses issues of navigation, content architecture and the user requirements.

SharePoint at DUT was initially implemented with specific purposes in mind and was primarily aimed at the IT department at the university. Its adoption subsequently grew and its use expanded into other avenues, most of which became apparent with its use. As we have seen, the library recognises that the tool has potential, however further investigation is necessary to establish the extent to which SharePoint could be seamlessly integrated with other systems including the library system. Lindvall, Rus and Sinha (2003: 139), as well as Holland and Dawson (2011: 400), suggest that an organisation may opt to adopt more than a single tool to address its KM needs. In the case of DUT Library, the survey results suggest that the library needs first to establish its KM needs, then fully understand the capability of SharePoint before deciding whether SharePoint is an appropriate tool for KM.

\section{References}

Aswath, L. and Gupta, S. 2009. Knowledge management tools and academic library services. International Conference on Academic Libraries (ICAL). 5-8 October 2009. Delhi, India. 187-192. [Online]. http://crl.du.ac.in/ical09/papers/index_files/ical-31_249_724_1_RV.pdf (26 July 2013).

Averweg, U. and Jory, S. 2007. An intranet as a strategic tool within a knowledge management strategy: reflecting on the evaluation of an intranet. Working paper 0011 (unpublished). [Online]. http://scholar.sun.ac.za/bitstream/handle/10019.1/4788/averweg_intranet_2007.pdf?sequence=1 (26 July 2013$)$.

Balmisse, G., Meingan, D. and Passerini, K. 2007. Technology trends in knowledge management tools. International Journal of Knowledge Management, 3(2): 118-131.

Beerkens, E. 2008. University policies for the Knowledge Society: global standardization, local reinvention. Perspectives on Global Development and Technology, 7(1): 15-36.

Edenius, M. and Borgerson, J. 2003. To manage knowledge by intranet. Journal of Knowledge Management, 7(5): 124136. 
Ennis, L. A. and Tims, R. S. 2010. Harnessing the power of SharePoint for library applications. Computers in Libraries, 30(5): 6-12.

Fenz, S. 2012. Increasing knowledge capturing efficiency by enterprise portals. VINE, 42(2): 237-250. DOI:10.1108/03055721211227264.

Fichter, D. 1999. Intranets: librarians, dive in! Online, 23(3): 107-108.

Guenther, K. 2003. Ten steps to intranet success. Online, 27(1): 66-69.

Herrera, K. 2008. From static files to collaborative workspace with SharePoint. Library Hi Tech, 26(1): 80-94.

Holland, S. and Dawson, R. 2011. Classification and selection of tools for quality knowledge management. Software Quality Journal, 19(2): 393-409.

Jedd, M. 2009. Taming SharePoint. Infonomics, 23(1): 34-38.

Koplowitz, R. and Owens, L. 2009. SharePoint: the backbone of your information architecture. KMWorld, 18(6). [Online]. http://www.kmworld.com (1 August 2013).

Kothari, C. R. 2004. Research methodology: methods and techniques. New Delhi: New Age International.

Leedy P. D. and Ormrod, J. E. 2013. Practical research: planning and design. $10^{\text {th }}$ ed. Upper Saddle River, N.J.: Pearson Education.

Lindvall, M., Rus, R. and Sinha, S. S. 2003. Software systems support for knowledge management. Journal of Knowledge Management, 7(5): 137-150.

Mphidi, H. and Snyman, R. 2004. The utilisation of an intranet as a knowledge management tool in academic libraries. The Electronic Library, 22(5): 393-400.

Perez, E. 1999. Knowledge management in the library - not. Database, 22(2): 75-78.

Rowley, J. 2000. Is higher education ready for knowledge management? International Journal of Educational Management, 14(7): 325-333.

Sarrafzadeh, M., Martin, B. and Hazeri, A. 2010. Knowledge management and its potential applicability for Libraries. Library Management, 31(3): 198-212.

Singh, Y. K. 2006. Fundamentals of research methodology and statistics. New Delhi: New Age International.

Weldon, L. S. J. 2010. The odd couple: SharePoint and librarians. LLRX.com (Law and Technology Resources for Legal Professionals). [Online]. http://www.Ilrx.com/features/SharePoint.htm (1 August 2013).

Yaacob, R. A., Jamaluddin, A. and Jusoff, K. 2010. Knowledge management and challenging roles of academic librarians. Management Science and Engineering, 4(4): 14-23.

Yaying, M. C. H. 2005. The implementation of knowledge management system in Taiwan's higher education. Journal of College Teaching \& Learning, 2(9): 35-42. 